\title{
A Bayesian analysis for the bivariate geometric distribution in the presence of covariates and censored data
}

Citation for published version (APA):

Davarzani, N., Achcar, J. A., Peeters, R., \& Smirnov, E. (2017). A Bayesian analysis for the bivariate geometric distribution in the presence of covariates and censored data. Journal of Statistics and Management Systems, 20(1), 1-16. https://doi.org/10.1080/09720510.2016.1160624

Document status and date:

Published: 01/01/2017

DOI:

10.1080/09720510.2016.1160624

Document Version:

Publisher's PDF, also known as Version of record

\section{Document license:}

Taverne

Please check the document version of this publication:

- A submitted manuscript is the version of the article upon submission and before peer-review. There can be important differences between the submitted version and the official published version of record.

People interested in the research are advised to contact the author for the final version of the publication, or visit the DOI to the publisher's website.

- The final author version and the galley proof are versions of the publication after peer review.

- The final published version features the final layout of the paper including the volume, issue and page numbers.

Link to publication

\footnotetext{
General rights rights.

- You may freely distribute the URL identifying the publication in the public portal. please follow below link for the End User Agreement:

www.umlib.nl/taverne-license

Take down policy

If you believe that this document breaches copyright please contact us at:

repository@maastrichtuniversity.nl

providing details and we will investigate your claim.
}

Copyright and moral rights for the publications made accessible in the public portal are retained by the authors and/or other copyright owners and it is a condition of accessing publications that users recognise and abide by the legal requirements associated with these

- Users may download and print one copy of any publication from the public portal for the purpose of private study or research.

- You may not further distribute the material or use it for any profit-making activity or commercial gain

If the publication is distributed under the terms of Article 25fa of the Dutch Copyright Act, indicated by the "Taverne" license above, 


\title{
A Bayesian analysis for the bivariate geometric distribution in the presence of covariates and censored data
}

\author{
Nasser Davarzani, Jorge Alberto Achcar, Ralf Peeters \& Evgueni Nikolaevich \\ Smirnov
}

To cite this article: Nasser Davarzani, Jorge Alberto Achcar, Ralf Peeters \& Evgueni Nikolaevich Smirnov (2017) A Bayesian analysis for the bivariate geometric distribution in the presence of covariates and censored data, Journal of Statistics and Management Systems, 20:1, 1-16, DOI: 10.1080/09720510.2016.1160624

To link to this article: http://dx.doi.org/10.1080/09720510.2016.1160624

Submit your article to this journal $\square$

山 Article views: 1

View related articles $\sqsubset$

View Crossmark data $\rtimes$ 


\title{
A Bayesian analysis for the bivariate geometric distribution in the presence of covariates and censored data
}

\author{
Nasser Davarzani * \\ Department of Data Science and Knowledge Engineering \\ Maastricht University \\ Maastricht \\ The Netherlands \\ Jorge Alberto Achcar ${ }^{+}$ \\ Departamento de Medicina Social, FMRP \\ Universidade de Sao Paulo Ribeirao Preto \\ Sao Paulo \\ Brazil \\ Ralf Peeters \\ Evgueni Nikolaevich Smirnov \\ Department of Data Science and Knowledge Engineering \\ Maastricht University \\ Maastricht \\ The Netherlands
}

\begin{abstract}
In this paper, we introduce a Bayesian analysis for bivariate geometric distributions applied to lifetime data in the presence of covariates and censored data using Markov Chain Monte Carlo (MCMC) methods. We show that the use of a discrete bivariate geometric distribution could bring us some computational advantages when compared to standard existing bivariate exponential lifetime distributions introduced in the literature assuming continuous lifetime data as for example, the exponential Block and Basu bivariate distribution. Posterior summaries of interest are obtained using the popular OpenBUGS software. A numerical illustration is introduced considering a medical data set related to the recurrence times of infection for kidney patients.
\end{abstract}

Keywords: Bivariate geometric distribution, Lifetime data, Bayesian analysis, MCMC methods, Covariates, Censored lifetimes.

${ }^{*}$ E-mail: n.davarzani@maastrichtuniversity.nl.

(C) If Taru Publications 


\section{Introduction}

Lifetime data is very common in medical and engineering applications associated to the survival times of patients or components. Usually, the lifetime denoted by $X$ is assumed to be a continuous random variable with a specified parametrical distribution as for example, an exponential, a Weibull, a log-normal or a gamma distribution (see for example [13]). In some cases we could have more than one lifetime related to each unit. As a special situation, we have two lifetimes $X_{1}$ and $X_{2}$ associated to each unit, where it is important to model the possible existing dependence between the lifetimes $X_{1}$ and $X_{2}$. This occurs for example in medical studies considering the failure times of paired organs like kidney, lungs, eyes, ears, dental implants amongmany others.Assuming continuous bivariate random variables we could consider different parametrical distributions to analyze bivariate lifetime data introduced in the literature [3,11, 12, 18, 20, 21].

Due to the fact that censoring is a key aspect of survival analysis, many works recently introduced in the literature have been related to the analysis of the bivariate lifetime distributions in the presence of right censoring, which most of them are in respect to the Marshall-Olkin bivariate distribution and Block and Basu distribution, (see for example, $[5,7,10,17,20])$. Despite the fact that discrete measuring of the lifetime values is so common in both medical and engineering experiments, we could see only few studies in this regard in the literature, (see for example $[6,9,22])$; however a bivariate discrete lifetime distribution in the presence of censoring may be of interest to discuss.

In applications, usually we have the presence of censoring data and covariates associated to each unit. In this situation, we could have some computational difficulties to get standard classical inferences for the parameters of these models, as for example, using maximum likelihood approach. In this way, the use of Bayesian methods is becoming very popular in the statistical analysis of bivariate lifetime models (see for example, $[1,19])$.

An alternative for the use of a continuous bivariate lifetime distribution, it is to assume that the lifetimes $X_{1}$ and $X_{2}$ are discrete random variables assuming any positive integer that is, we round the lifetimes given with decimal part to an integer. In this way, [2] introduced a general multivariate geometric distribution which showed that it leads in a natural way to the Marshall-Olkin multivariate exponential distribution. [16] studied the characterizations of the bivariate exponential and geometric distributions. The bivariate geometric distribution proposed by [2] has joint probability mass function ( $p m f)$ given by, 


$$
P\left(X_{1}, X_{2}\right)= \begin{cases}P_{1}\left(x_{1}, x_{2}\right)=\theta_{1} \theta_{2}\left(1-\theta_{1}-\theta_{2}\right)^{x_{1}-1}\left(1-\theta_{2}\right)^{x_{2}-x_{1}-1}, & x_{1}<x_{2} \\ 0 & x_{1}=x_{2} \\ P_{2}\left(x_{1}, x_{2}\right)=\theta_{1} \theta_{2}\left(1-\theta_{1}-\theta_{2}\right)^{x_{2}-1}\left(1-\theta_{1}\right)^{x_{1}-x_{2}-1}, & x_{1}>x_{2}\end{cases}
$$

where the marginal probability mass function for $X_{1}$ and $X_{2}$ are standard geometric distributions starting at one, given respectively by,

$$
p\left(x_{1}\right)=\left(1-\theta_{1}\right)^{x_{1}-1} \theta_{1}, x_{1}=1,2,3, \ldots,
$$

and,

$$
p\left(x_{2}\right)=\left(1-\theta_{2}\right)^{x_{2}-1} \theta_{2}, x_{2}=1,2,3, \ldots,
$$

with means, variances, covariance and correlation given respectively, by

$$
\begin{gathered}
\mu_{1}=E\left(X_{1}\right)=\frac{1}{\theta_{1}}, \mu_{2}=E\left(X_{2}\right)=\frac{1}{\theta_{2}} \\
\sigma_{1}^{2}=\operatorname{Var}\left(X_{1}\right)=\frac{1-\theta_{1}}{\theta_{1}^{2}}, \sigma_{2}^{2}=\operatorname{Var}\left(X_{2}\right)=\frac{1-\theta_{2}}{\theta_{2}^{2}}, \\
\operatorname{Cov}\left(X_{1}, X_{2}\right)=\frac{-1}{1-r}, \\
\rho_{12}=\operatorname{Corr}\left(X_{1}, X_{2}\right)=-\frac{\theta_{1} \theta_{2}}{(1-r)\left[\left(1-\theta_{1}\right)\left(1-\theta_{2}\right)\right]^{0.5}},
\end{gathered}
$$

where, $r=1-\theta_{1}-\theta_{2}$.

From (1), we observe that this bivariate discrete model assumes that $P\left(X_{1}=X_{2}\right)=0$, but in applications we could have this probability very small, but different of zero, a restrictive property of the model. In some cases, however, like studying the time of blindness of eyes or infection of kidneys, this probability practically is zero. In such cases the proposed model will be more fit to the data rather that the models with positive probability of $X_{1}=X_{2}$. In medical or engineering applications, we usually consider a discretization of continuous data and we should choose an appropriate scale to use in our data. For example, if we have continuous observations 5.2 and 5.3 we could use 52 and 53 (multiplying by 10) in place of using the scale rounding up the two values equal to 5. Other characteristic of this distribution, is the negative covariance structure that is linked to the genesis of the distribution (see Arnold,1975). 
In this paper, we explore the use of a bivariate geometric distribution to analyze bivariate lifetime data, in the presence of censoring. This paper is organized as follows: in section 2, we consider the bivariate lifetime variables under the right censoring scheme and we drive the likelihood; in section 3, we introduce a Bayesian analysis in the presence of censored data; in section 4, we consider a numerical illustration considering the analysis of recurrence times of infection for kidney patients; finally in section 5, we introduce some concluding remarks.

\section{Preliminaries}

Let $\left(X_{11}, X_{21}\right), \ldots,\left(X_{1 n}, X_{2 n}\right)$ be a random sample of size $n$ from an ordinary bivariate geometric $(O B G)$ distribution with joint $(p m f)$ in (1). Suppose that either $X_{1}$ or $X_{2}$ can be censored at $Y_{1}$ or $Y_{1}$, respectively and that censoring is independent of the lifetimes. Let us subdivide the $n$ observations into four classes:

$C_{1}: X_{1 i}<Y_{1 i}$ and $X_{2 i}<Y_{2 i}$ thus both $x_{1 i}$ or $x_{2 i}$ are the real lifetimes;

$C_{2}: X_{1 i}<Y_{1 i}$ and $Y_{2 i}<X_{2 i}$, that is, we observe $x_{1 i}$ and $y_{2 i}$;

$C_{3}: Y_{1 i}<X_{1 i}$ and $X_{2 i}<Y_{2 i}$, we observe $y_{1 i}$ and $x_{2 i}$;

$C_{3}: Y_{1 i}<X_{1 i}$ and $Y_{2 i}<X_{2 i i^{\prime}}$ we observe $y_{1 i}$ and $y_{2 i}$;

Suppose $I_{1}=\left\{i \in C_{1} \mid x_{1 i}<x_{2 i}\right\}, I_{2}=\left\{i \in C_{1} \mid x_{2 i}<x_{1 i}\right\}, I_{3}=\left\{i \in C_{2} \mid x_{1 i}<x_{2 i}\right\}$, $I_{4}=\left\{i \in C_{2} \mid x_{2 i}<x_{1 i},\right\}, I_{5}=\left\{i \in C_{3} \mid x_{1 i}<x_{2 i}\right\}, I_{6}=\left\{i \in C_{3} \mid x_{2 i}<x_{1 i}\right\}, I_{7}=$ $\left\{i \in C_{4} \mid x_{1 i}<x_{2 i},\right\}, I_{8}=\left\{i \in C_{4} \mid x_{2 i}<x_{1 i}\right\}$

Now based on the aforementioned definitions, the likelihood function of the actual observed data considering bivariate right censored lifetimes in this model is given by,

$$
\begin{aligned}
& \prod_{i \in I_{6}}\left(\sum_{x_{i i}=y_{1 i}+1}^{\infty} P_{2}\left(x_{1 i}, x_{2 i}\right)\right) \prod_{i \in I_{7}}\left(\sum_{x_{1 i}=y_{1 i}+1 x_{2 i}=y_{2 i}+1}^{\infty} P_{1}\left(x_{1 i}, x_{2 i}\right)\right) \\
& \prod_{i \in I_{8}}\left(\sum_{x_{1 i}=y_{1 i}+1 x_{2 i}=y_{2 i}+1}^{\infty} P_{2}\left(x_{1 i}, x_{2 i}\right)\right) \\
& \prod_{i \in I_{6}}\left(\sum_{x_{1 i}=y_{1 i}+1}^{\infty} P_{2}\left(x_{1 i}, x_{2 i}\right)\right) \prod_{i \in I_{7}}\left(\sum_{x_{1 i}=y_{1 i}+1 x_{2 i}=y_{2 i}+1}^{\infty} P_{1}^{\infty}\left(x_{1 i}, x_{2 i}\right)\right) \\
& \prod_{i \in I_{8}}\left(\sum_{x_{1 i}=y_{1 i}+1 x_{2 i}=y_{2 i}+1}^{\infty} P_{2}\left(x_{1 i}, x_{2 i}\right)\right)
\end{aligned}
$$


where,

$$
\begin{aligned}
& \sum_{x_{2 i}=y_{2 i}+1}^{\infty} P_{1}\left(x_{1 i}, x_{2 i}\right)=\theta_{1}\left(1-\theta_{1}-\theta_{2}\right)^{x_{1 i}-1}\left(1-\theta_{2}\right)^{y_{2 i}-x_{1 i}-1}, \\
& \sum_{x_{2 i}=y_{2 i}+1}^{\infty} P_{2}\left(x_{1 i}, x_{2 i}\right)=\theta_{1}\left(1-\theta_{1}-\theta_{2}\right)^{y_{2 i}}\left(1-\theta_{1}\right)^{x_{1 i}-y_{2 i}-1}, \\
& \sum_{x_{1 i}=y_{1 i}+1}^{\infty} P_{1}\left(x_{1 i}, x_{2 i}\right)=\theta_{2}\left(1-\theta_{2}\right)^{x_{2 i}-y_{1 i}-1}\left(1-\theta_{1}-\theta_{2}\right)^{y_{1 i}}, \\
& \sum_{x_{1 i}=y_{1 i}+1}^{\infty} P_{2}\left(x_{1 i}, x_{2 i}\right)=\theta_{2}\left(1-\theta_{1}\right)^{y_{1 i}-x_{2 i}}\left(1-\theta_{1}-\theta_{2}\right)^{x_{2 i}-1},
\end{aligned}
$$

and

$$
\sum_{x_{1 i}=y_{1 i}+1 x_{2 i}=y_{2 i}+1}^{\infty} P_{2}\left(x_{1 i}, x_{2 i}\right)=\left(1-\theta_{1}\right)^{y_{1 i}-y_{2 i}}\left(1-\theta_{1}-\theta_{2}\right)^{y_{2 i}} .
$$

We can summarize the likelihood function as follow:

$$
L\left(\theta_{1}, \theta_{2}\right)=\theta_{1}^{m_{1}} \theta_{2}^{m_{2}}\left(1-\theta_{1}\right)^{z_{1}}\left(1-\theta_{2}\right)^{z_{2}}\left(1-\theta_{1}-\theta_{2}\right)^{z_{12}},
$$

where, $n_{i}=\left|I_{i}\right|, i=1, \ldots, 8$, be the cardinal number of the set $I_{i}, m_{1}=n_{1}+n_{2}$ $+n_{3}+n_{4}, m_{2}=n_{1}+n_{2}+n_{5}+n_{6}, z_{12}=\sum_{i \in I_{1} \cup I_{3}} x_{1 i}+\sum_{i \in I_{2} \cup I_{6}} x_{2 i}+\sum_{i \in I_{5} \cup I_{7}} y_{1 i}+\sum_{i \in I_{4} \cup I_{8}} y_{2 i}$, $z_{1}=\sum_{i \in I_{2} \cup I_{4}} x_{1 i}-\sum_{i \in I_{2} \cup I_{6}} x_{2 i}+\sum_{i \in I_{6} \cup I_{8}} y_{1 i}-\sum_{i \in I_{4} \cup I_{8}} y_{2 i}$ and $z_{2}=\sum_{i \in I_{1}} x_{2 i}-\sum_{i \in I_{1} \cup I_{3}} x_{1 i}+\sum_{i \in I_{3} \cup I_{5} \cup I_{7}} y_{2 i}$ $-\sum_{i \in I_{5} \cup I_{7}} y_{1 i}$.

\section{A Bayesian Analysis in the Presence of Covariates and Censored Observations}

In this section we consider the Bayes estimators of $\theta_{1}, \theta_{2}$ and the survival functions under the Squared error loss (SEL) function. In this way, we are able to obtain the closed form estimators for all parameters. Suppose that $\left(\theta_{1}, \theta_{2}\right)$ has the following prior density:

$$
\pi\left(\theta_{1}, \theta_{2}\right) \propto \theta_{1}^{\alpha_{1}-1} \theta_{2}^{\alpha_{2}-1}\left(1-\theta_{1}-\theta_{2}\right)^{\alpha_{\circ}-1}, \theta_{1}+\theta_{2}<1,
$$

which is a conjugate prior, denoted by $\operatorname{Dir}_{2}\left(\alpha_{0}, \alpha_{1}, \alpha_{2}\right)$, i.e., the Dirichlet distribution with parameters $\alpha_{0}, \alpha_{1}$ and $\alpha_{2}$. Combining the likelihood 
function (2) with the prior (3), the joint posterior density function of $\theta_{1}$ and $\theta_{2}$ can be written as

$$
\pi\left(\theta_{1}, \theta_{2} \mid x, y\right) \propto \theta_{1}^{m_{1}+\alpha_{1}-1} \theta_{2}^{m_{2}+\alpha_{2}-1}\left(1-\theta_{1}\right)^{z_{1}}\left(1-\theta_{2}\right)^{z_{2}}\left(1-\theta_{1}-\theta_{2}\right)^{z_{12}+\alpha_{0}-1} .
$$

Using the result,

$$
\left(1-\theta_{i}\right)^{z_{i}}=\sum_{r=0}^{\infty}\left(\begin{array}{c}
z_{i} \\
r
\end{array}\right) \theta_{i}^{r}, i=1,2,
$$

we can rewrite (4) as:

$$
\begin{aligned}
\pi\left(\theta_{1}, \theta_{2} \mid x, y\right)=\frac{1}{D_{1}} \sum_{r=0}^{\infty} \sum_{k=0}^{\infty}(-1)^{r+k}\left(\begin{array}{c}
z_{1} \\
r
\end{array}\right)\left(\begin{array}{c}
z_{2} \\
k
\end{array}\right) \theta_{1}^{m_{1}+\alpha_{1}+r-1} \\
\theta_{2}^{m_{2}+\alpha_{2}+k-1}\left(1-\theta_{1}-\theta_{2}\right)^{z_{12}+\alpha_{0}-1},
\end{aligned}
$$

where,

$$
D_{1}=\sum_{r=0}^{\infty} \sum_{k=0}^{\infty} \frac{(-1)^{r+k}\left(\begin{array}{c}
z_{1} \\
r
\end{array}\right)\left(\begin{array}{c}
z_{2} \\
k
\end{array}\right) \Gamma\left(m_{1}+m_{2}+\alpha_{0}+\alpha_{1}+\alpha_{2}+z_{12}+r+k\right)}{\Gamma\left(m_{1}+\alpha_{1}+r\right) \Gamma\left(m_{2}+\alpha_{2}+k\right) \Gamma\left(z_{12}+\alpha_{0}\right)} .
$$

Now, the Bayes estimators for $\theta_{1}, \theta_{2}$ and the marginal survival functions of $X_{1}$ and $X_{2}$, under the SEL function are obtained, respectively as follow:

$$
\begin{gathered}
E\left(\theta_{1} \mid \boldsymbol{x}, \boldsymbol{y}\right)= \begin{cases}\frac{m_{1}+\alpha_{1}}{m_{1}+m_{2}+\alpha_{0}+\alpha_{1}+\alpha_{2}+z_{12}} & \text { if } z_{1}=z_{2}=0 \\
\sum_{r=0}^{\infty} \sum_{k=0}^{\infty}(-1)^{r+k}\left(\begin{array}{c}
z_{1} \\
r
\end{array}\right)\left(\begin{array}{c}
z_{2} \\
k
\end{array}\right) \frac{\Gamma\left(m_{1}+\alpha_{1}+r+1\right) \Gamma\left(m_{2}+\alpha_{2}+k\right)}{\Gamma\left(m_{1}+m_{2}+\alpha_{0}+\alpha_{1}+\alpha_{2}+z_{12}+r+k+1\right)} & \text { if } z_{1}, z_{2} \neq 0, \\
\sum_{r=0}^{\infty} \sum_{k=0}^{\infty}(-1)^{r+k}\left(\begin{array}{c}
z_{1} \\
r
\end{array}\right)\left(\begin{array}{c}
z_{2} \\
k
\end{array}\right) \frac{\Gamma\left(m_{1}+\alpha_{1}+r\right) \Gamma\left(m_{2}+\alpha_{2}+k\right)}{\Gamma\left(m_{1}+m_{2}+\alpha_{0}+\alpha_{1}+\alpha_{2}+z_{12}+r+k\right)} & \text { if } z_{1}=z_{2}=0\end{cases} \\
E\left(\theta_{2} \mid \boldsymbol{x}, \boldsymbol{y}\right)= \begin{cases}\frac{m_{1}+\alpha_{1}}{m_{1}+m_{2}+\alpha_{0}+\alpha_{1}+\alpha_{2}+z_{12}} \\
\sum_{r=0}^{\infty} \sum_{k=0}^{\infty}(-1)^{r+k}\left(\begin{array}{c}
z_{1} \\
r
\end{array}\right)\left(\begin{array}{c}
z_{2} \\
k
\end{array}\right) \frac{\Gamma\left(m_{1}+\alpha_{1}+r\right) \Gamma\left(m_{2}+\alpha_{2}+k+1\right)}{\Gamma\left(m_{1}+m_{2}+\alpha_{0}+\alpha_{1}+\alpha_{2}+z_{12}+r+k+1\right)} \\
\sum_{r=0}^{\infty} \sum_{k=0}^{\infty}(-1)^{r+k}\left(\begin{array}{c}
z_{1} \\
r
\end{array}\right)\left(\begin{array}{c}
z_{2} \\
k
\end{array}\right) \frac{\Gamma\left(m_{1}+\alpha_{1}+r\right) \Gamma\left(m_{2}+\alpha_{2}+k\right)}{\Gamma\left(m_{1}+m_{2}+\alpha_{0}+\alpha_{1}+\alpha_{2}+z_{12}+r+k\right)} & \text { if } z_{1}, z_{2} \neq 0,\end{cases}
\end{gathered}
$$




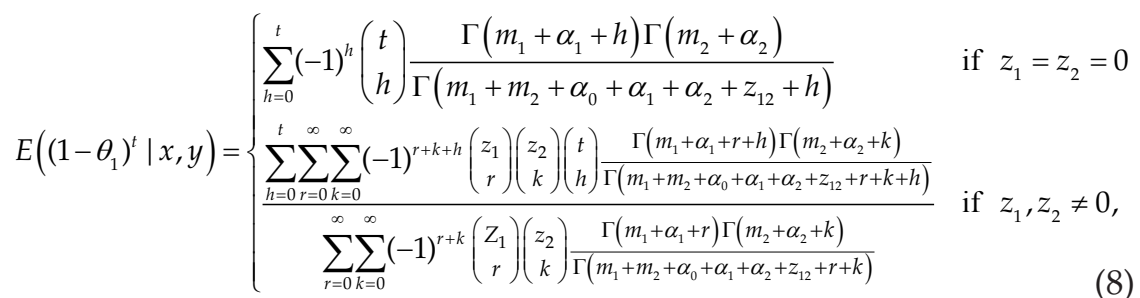

and,

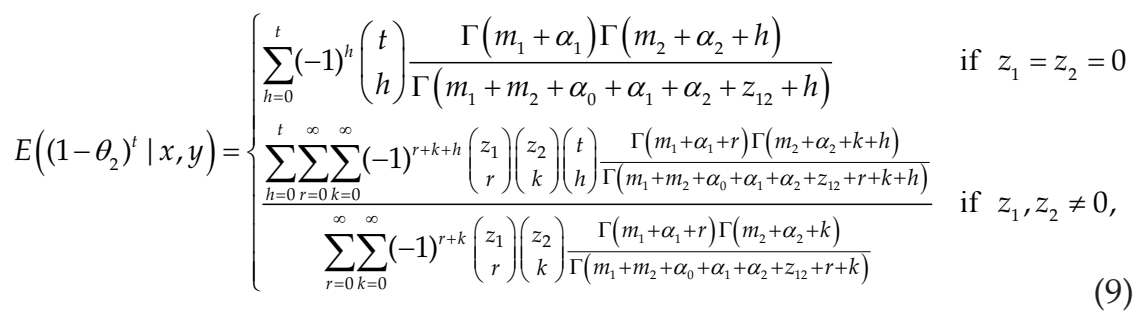

where $t$ is a positive real number and

$$
\left(\begin{array}{l}
t \\
i
\end{array}\right)= \begin{cases}\frac{t(t-1) \ldots(t-i+1)}{i !} & i=1,2, \ldots \\
1 & i=0 .\end{cases}
$$

Using the above exact expressions we could have some computational difficulties to obtain the Bayesian estimates in applications. As an alternative to find accurate posterior summaries of interest, we could use MCMC methods (see for example $[4,8]$ ). To this end, we write the log-likelihood function in a setting which it is easier to apply in existing available MCMC softwares used to get the posterior summaries of interesting, like the OpenBugs software.

$$
\begin{aligned}
l\left(\theta_{1}, \theta_{2}\right)= & \sum_{i=1}^{n} v_{i} \delta_{1 i} \delta_{2 i} \log P_{1}\left(x_{1 i}, x_{2 i}\right)+\sum_{i=1}^{n}\left(1-v_{i}\right) \delta_{1 i} \delta_{2 i} \log P_{2}\left(x_{1 i}, x_{2 i}\right) \\
& +\sum_{i=1}^{n} v_{i} \delta_{1 i}\left(1-\delta_{2 i}\right) \log \left(\sum_{x_{2 i}=y_{2 i}+1}^{\infty} P_{1}\left(x_{1 i}, x_{2 i}\right)\right) \\
& +\sum_{i=1}^{n}\left(1-v_{i}\right) \delta_{1 i}\left(1-\delta_{2 i}\right) \log \left(\sum_{x_{2 i}=y_{2 i}+1}^{\infty} P_{2}\left(x_{1 i}, x_{2 i}\right)\right) \\
& +\sum_{i=1}^{n} v_{i}\left(1-\delta_{1 i}\right) \delta_{2 i} \log \left(\sum_{x_{1 i}=y_{1 i}+1}^{\infty} P_{1}\left(x_{1 i}, x_{2 i}\right)\right) \\
& +\sum_{i=1 n}^{n}\left(1-v_{i}\right)\left(1-\delta_{1 i}\right) \delta_{2 i} \log \left(\sum_{x_{1 i}=y_{1 i}+1}^{\infty} P_{2}\left(x_{1 i}, x_{2 i}\right)\right)
\end{aligned}
$$




$$
\begin{aligned}
& +\sum_{i=1}^{n} v_{i}\left(1-\delta_{1 i}\right)\left(1-\delta_{2 i}\right) \log \left(\sum_{x_{1 i}=y_{1 i}+1 x_{2 i}=y_{2 i}+1}^{\infty} P_{1}\left(x_{1 i}, x_{2 i}\right)\right) \\
& +\sum_{i=1}^{n}\left(1-v_{i}\right)\left(1-\delta_{1 i}\right)\left(1-\delta_{2 i}\right) \log \left(\sum_{x_{1 i}=y_{1 i}+1 x_{2 i}=y_{2 i}+1}^{\infty} P_{2}\left(x_{1 i}, x_{2 i}\right)\right),
\end{aligned}
$$

where $\delta_{1 i}, \delta_{1 i}$ and $v_{i}$ are indicator variables, given by, $\delta_{j i}=1$ if $X_{j i}$ is an observed lifetime; $\delta_{j i}=0$ if $X_{j i}$ is a censored observation, for $j=1,2 ; i=1,2$, $\ldots, n$, and $v_{i}=1$ if $x_{1 i}<x_{2 i}$ and $v_{i}=0$ if $x_{1 i} \geq x_{2 i}$.

In the presence of a covariate vector $u=\left(u_{1}, u_{2}, \ldots, u_{p}\right)$ associated to each bivariate lifetime $\left(X_{1}, X_{2}\right)$, we could assume the following logistic regression models:

$$
\begin{aligned}
& \theta_{1 i}=\frac{\exp \left\{\beta_{1}^{\prime} u_{i}\right\}}{1+\exp \left\{\beta_{1}^{\prime} u_{i}\right\}}, \\
& \theta_{2 i}=\frac{\exp \left\{\beta_{2}^{\prime} u_{i}\right\}}{1+\exp \left\{\beta_{2}^{\prime} u_{i}\right\}},
\end{aligned}
$$

where, $\beta_{j}^{\prime}=\left(\beta_{j 1}, \beta_{j 2}, \ldots, \beta_{j p}\right), j=1,2$, is the regression parameter vector and $u_{i}=\left(u_{1 i}, u_{2 i}, \ldots, u_{p i}\right), i=1, \ldots n$.

For a Bayesian analysis of the model, we assume normal prior distributions for the regression parameters, that is:

$$
\beta_{k l} \sim N\left(0, \sigma_{k l}^{2}\right)
$$

where $k=1,2$ and $l=1,2, \ldots, p$. We also assume prior independence among the regression parameters and that the hyperparameter $\sigma_{k l}^{2}$ is known.

\section{An application considering the recurrence times of infection for kidney patients}

In this section, we present as a numerical illustration of our proposed methodology, the analysis of a bivariate lifetime data set introduced by [15] assuming the bivariate geometric distribution. This data set is related to kidney infection where the recurrence of infection of 38 kidney patients, using portable dialysis machines, are recorded. Infections may occur at the location of insertion of the catheter. The time recorded, called infection time, is either the survival time (in days) of the patient until an infection occurred and the catheter had to be removed, or the censored time, where the catheter was removed by others reasons. The catheter is reinserted after 
Table 1

Recurrence times of infections in 38 kidney patients.

\begin{tabular}{|c|c|c|c|c|c|}
\hline Patient & First time & $\begin{array}{l}\text { Second } \\
\text { time }\end{array}$ & $\begin{array}{c}\text { Censoring first } \\
\text { time }\end{array}$ & $\begin{array}{l}\text { Censoring } \\
\text { second time }\end{array}$ & Sex \\
\hline 1 & 8 & 16 & 1 & 1 & 1 \\
\hline 2 & 23 & 13 & 1 & 0 & 2 \\
\hline 3 & 22 & 28 & 1 & 1 & 1 \\
\hline 4 & 447 & 318 & 1 & 1 & 2 \\
\hline 5 & 30 & 12 & 1 & 1 & 1 \\
\hline 6 & 24 & 245 & 1 & 1 & 2 \\
\hline 7 & 7 & 9 & 1 & 1 & 1 \\
\hline 8 & 511 & 30 & 1 & 1 & 2 \\
\hline 9 & 53 & 196 & 1 & 1 & 2 \\
\hline 10 & 15 & 154 & 1 & 1 & 1 \\
\hline 11 & 7 & 333 & 1 & 1 & 2 \\
\hline 12 & 141 & 8 & 1 & 0 & 2 \\
\hline 13 & 96 & 38 & 1 & 1 & 2 \\
\hline 14 & 149 & 70 & 0 & 0 & 2 \\
\hline 15 & 536 & 25 & 1 & 0 & 2 \\
\hline 16 & 17 & 4 & 1 & 0 & 1 \\
\hline 17 & 185 & 117 & 1 & 1 & 2 \\
\hline 18 & 292 & 114 & 1 & 1 & 2 \\
\hline 19 & 22 & 159 & 0 & 0 & 2 \\
\hline 20 & 15 & 108 & 1 & 0 & 2 \\
\hline 21 & 152 & 562 & 1 & 1 & 1 \\
\hline 22 & 402 & 24 & 1 & 0 & 2 \\
\hline 23 & 13 & 66 & 1 & 1 & 2 \\
\hline 24 & 39 & 46 & 1 & 0 & 2 \\
\hline 25 & 12 & 40 & 1 & 1 & 1 \\
\hline 26 & 113 & 201 & 0 & 1 & 2 \\
\hline 27 & 132 & 156 & 1 & 1 & 2 \\
\hline 28 & 34 & 30 & 1 & 1 & 2 \\
\hline 29 & 2 & 25 & 1 & 1 & 1 \\
\hline 30 & 130 & 26 & 1 & 1 & 2 \\
\hline 31 & 27 & 58 & 1 & 1 & 2 \\
\hline 32 & 5 & 43 & 0 & 1 & 2 \\
\hline 33 & 152 & 30 & 1 & 1 & 2 \\
\hline 34 & 190 & 5 & 1 & 0 & 2 \\
\hline 35 & 119 & 8 & 1 & 1 & 2 \\
\hline 36 & 54 & 16 & 0 & 0 & 2 \\
\hline 37 & 6 & 78 & 0 & 1 & 2 \\
\hline 38 & 63 & 8 & 1 & 0 & 1 \\
\hline
\end{tabular}

Censoring (0); infection occurrence (1); male (1); female (2). 
some time and the second infection time is again observed or censored (data set in Table 1).

In the statistical analysis of the recurrence times of infections (see data set in Table 1), let us assume the bivariate geometric distribution with density (1) assuming discrete times. As a first analysis, we do not consider the presence of the covariate Sex. Let us denote this model as "model 1 ". For a Bayesian analysis of "model 1", let us assume a Dirichlet prior distribution (3) for $\theta_{1}$ and $\theta_{2}$ with hyperparameter values $\alpha_{1}=\alpha_{2}=\alpha_{3}=1$.

Bayesian estimators for the parameters of the model were obtained from simulated samples of the joint posterior distribution of interest using the OpenBUGS software (Spiegelhalter et al, 2003). In the simulation approach, we discarded the first 10,000 simulated Gibbs samples ("burnin-samples") to eliminate the effect of the initial values for the parameters of the model. Choosing every 50th simulated Gibbs sample, we obtained a final sample of size 1,000 to get the posterior summaries of interest. Convergence of the Gibbs sampling algorithm was monitored using existing methods as time series plots for the simulated samples.

The posterior means, the posterior standard-deviations and 95\% credible intervals for the parameters $\theta_{1}, \theta_{2}$ and $r$ are given in Table 2. We also have in Table 2, the posterior summaries for the means, standarddeviations and for thecorrelation coefficient (see (1)) for the lifetimes $X_{1}$ and $X_{2}$.

To have some idea of the fit of the proposed model, we could compare sample means and sample standard-deviations based on the

Table 2

Posterior summaries "model 1".

\begin{tabular}{c|l|l|l}
\hline Parameter & \multicolumn{1}{|c|}{ Mean } & \multicolumn{1}{c|}{ S.D. } & \multicolumn{1}{c}{$95 \%$ Credible interval } \\
\hline$\mu_{1}$ & 133.4 & 23.95 & $(96.28 ; 186.9)$ \\
$\mu_{2}$ & 131.7 & 27.97 & $(89.44 ; 201.8)$ \\
$r$ & 0.9844 & 0.002079 & $(0.9802 ; 0.9883)$ \\
$\sigma_{1}$ & 132.9 & 23.95 & $(95.78 ; 186.4)$ \\
$\sigma_{2}$ & 131.2 & 27.97 & $(88.93 ; 201.3)$ \\
$\theta_{1}$ & 0.007728 & 0.00133 & $(0.005389 ; 0.0104)$ \\
$\theta_{2}$ & 0.007918 & 0.001607 & $(0.004963 ; 0.01119)$ \\
$\rho_{12}$ & -0.003873 & 0.0526 & $(-0.00493 ;-0.002902)$ \\
\hline
\end{tabular}


uncensored observations with the Bayesian estimators obtained from the simulated Gibbs samples. In this way, we have the sample means and sample standard deviations for the first (32 uncensored observations) and second times (26 uncensored observations) given by: sample mean for first infection $=121.80$; sample mean for second infection $=113.50$; standard deviation for first infection $=153.30$ and standard deviation for second infection $=131.40$. Comparing these values with the obtained results of Table 2 (Monte Carlo estimates for the posterior means and 95\% credible intervals for $\mu_{1}, \mu_{2}, \sigma_{1}$ and $\sigma_{2}$ ), we have an indication of good fit of the geometric distribution with density (1) for the recurrence times of infection for kidney patients introduced in Table 1.

In the presence of the covariate Sex, we assume the logistic regression model (11) introduced in Section 2 for the parameters $\theta_{1}, \theta_{2}, \sigma_{1}$ and $\sigma_{2}$, that is

$$
\begin{aligned}
& \log i t\left(\theta_{1 i}\right)=\beta_{10}+\beta_{11} \operatorname{Sex}_{i} \\
& \operatorname{logit}\left(\theta_{2 i}\right)=\beta_{20}+\beta_{21} \operatorname{Sex}_{i},
\end{aligned}
$$

where $i=1,2, \ldots, 18 ; \operatorname{logit}(z)=\log [z /(1-z)], \operatorname{Sex}_{i}=1 \quad$ (male) and $\operatorname{Sex}_{i}=0$ (female). Let us denote this model as "model 2". Observe that $r_{i}=\theta_{1 i}-\theta_{2 i}$.

Assuming normal prior distributions $N(0,10)$, for the regression parameters $\beta_{j 0}, \mathrm{j}=1,2$, and normal prior distributions $N(0,1)$ for the regression parameters $\beta_{j 1}, \mathrm{j}=1,2$, where $N\left(a, b^{2}\right)$ denotes a normal distribution with mean equals to a and variance equals to $b^{2}$, and also using the OpenBUGS software considering a burn-in-sample of size 10,000 and taking every $50^{\text {th }}$ sample, after this burn-in-sample period, we have in Table 3, the posterior summaries of interest for "model 2", obtained using a final Gibbs samples of size 1,000.

Table 3

Posterior summaries "model 2".

\begin{tabular}{c|l|l|l}
\hline Parameter & \multicolumn{1}{|c|}{ Mean } & \multicolumn{1}{|c|}{ S.D. } & \multicolumn{1}{c}{$95 \%$ Credible interval } \\
\hline$\beta_{10}$ & -5.126 & 0.2069 & $(-5.55 ;-4.73)$ \\
$\beta_{11}$ & 1.441 & 0.3765 & $(0.69 ; 2.154)$ \\
$\beta_{20}$ & -4.951 & 0.2331 & $(-5.423 ;-4.509)$ \\
$\beta_{21}$ & 0.2064 & 0.3892 & $(-0.639 ; 0.9607)$ \\
\hline
\end{tabular}


From the results of Table 3 , we observe that zero is included in the $95 \%$ credible interval for $\beta_{21}$ but not for $\beta_{11}$, that is, the lifetime $X_{2}$ is not affected by the covariate Sex, but $X_{1}$ is affected by the covariate Sex.

It is interesting to observe that the data set introduced by [15] related to the recurrence times of infection of 38 kidney patients also have other four covariates besides the covariate sex: age, presence of disease type GN, presence of disease type AN, presence of disease type PKD, where GN, AN and PKD are short forms of Glomerulo Neptirits, acute Neptirits and Polyciatic kidney disease.

In the presence of all five covariates, we assume the logistic regression model (11) introduced in Section 2 for the parameters $\theta_{1}$ and $\theta_{2}$, that is,

$$
\begin{aligned}
& \operatorname{logit}\left(\theta_{1 i}\right)=\beta_{10}+\beta_{11} \operatorname{Sex}_{i}+\beta_{12} \text { Age }_{i}+\beta_{13} G N_{i}+\beta_{14} A N_{i}+\beta_{15} P K D_{i} \\
& \operatorname{logit}\left(\theta_{2 i}\right)=\beta_{20}+\beta_{21} \operatorname{Sex}_{i}+\beta_{22} \text { Age }_{i}+\beta_{23} G N_{i}+\beta_{24} A N_{i}+\beta_{25} P K D_{i} .
\end{aligned}
$$

Let us denote this model as "model 3". Using some prior information based on a preliminary analysis considering independent lifetimes (use of empirical Bayesian methods), we assume the following priors for the regression parameters: $\beta_{10} \sim N(-1.4,1), \beta_{11} \sim N(-2.1,1), \beta_{12} \sim N(0.008,10)$, $\beta_{13} \sim N(0.22,1), \quad \beta_{14} \sim N(0.76,1), \quad \beta_{15} \sim N(-1.3,1), \quad \beta_{20} \sim N(-3.2,1)$, $\beta_{21} \sim N(-0.9,1), \beta_{22} \sim N(0.002,10), \beta_{23} \sim N(-0.98,1), \beta_{24} \sim N(-0.044,10)$, $\beta_{25} \sim N(-1.34,1)$. This choice of informative priors improves the convergence of the Gibbs sampling algorithm using the OpenBUGS software. Using the OpenBUGS software also considering a "burn-in-sample" of size 10,000 and generating a final Gibbs sample of size 1,000 taking every $50^{\text {th }}$ sample, we have in Table 4, the posterior summaries of interest.

From the results of Table 4 , we observe that,

(a) Lifetime $X_{1}$

Covariates Sex and disease type PKD are significative (zero is not included in the $95 \%$ credible intervals $(-2.854 ;-1.664)$ for $\beta_{11}$ and $(-2.551 ;-0.1825)$ for $\left.\beta_{15}\right)$ all other covariates are not significative (zero is included in the $95 \%$ credible intervals for the regression parameters $\beta_{12}, \beta_{13}$ and $\beta_{14}$ ).

(b) Lifetime $X_{2}$

Covariates Sex and disease type PKD are significative (zero is not included in the $95 \%$ credible intervals $(-1.719 ;-0.2097)$ for $\beta_{21}$ and $(-$ $2.504 ;-0.3823$ ) for $\beta_{25}$ ) all other covariates are not significative (zero is included in the $95 \%$ credible intervals for the regression parameters $\beta_{22}, \beta_{23}$ and $\beta_{24}$ ). 
Table 4

Posterior summaries "model 3".

\begin{tabular}{c|l|l|l}
\begin{tabular}{c|c} 
Parameter \\
$\beta_{10}$
\end{tabular} & \multicolumn{1}{|c|}{ Mean } & \multicolumn{1}{|c|}{ S.D. } & \multicolumn{1}{|c}{$95 \%$ Credible interval } \\
$\beta_{11}$ & -2.245 & 0.6043 & $(-2.421 ;-0.1361)$ \\
$\beta_{12}$ & 0.007145 & 0.3118 & $(-2.854 ;-1.664)$ \\
$\beta_{13}$ & 0.2813 & 0.01228 & $(-0.01694 ; 0.0315)$ \\
$\beta_{14}$ & 0.8213 & 0.4522 & $(-0.5533 ; 1.105)$ \\
$\beta_{15}$ & -1.358 & 0.6021 & $(-0.07333 ; 1.675)$ \\
$\beta_{20}$ & -3.134 & 0.7639 & $(-2.551 ;-0.1825)$ \\
$\beta_{21}$ & -0.9501 & 0.3828 & $(-4.581 ;-1.758)$ \\
$\beta_{22}$ & 0.002388 & 0.01263 & $(-0.02311 ; 0.02737)$ \\
$\beta_{23}$ & -0.2642 & 0.4617 & $(-1.184 ; 0.5899)$ \\
$\beta_{24}$ & 0.02223 & 0.27 \\
$\beta_{25}$ & -1.442 & 0.5549 & $(-0.4982 ; 0.5527)$ \\
\hline
\end{tabular}

As a comparison for the three proposed models, we could use a Bayesian discrimination criterion, as for example, the DIC (Deviance Information Criterion) introduced by [23], (see the appendix) and given automatically by the OpenBUGS software. Assuming "model 1", that is, the bivariate geometric distribution not considering the presence of covariates, we have a Monte Carlo estimate for DIC given by the value 685.9; assuming "model 2", that is, the bivariate geometric distribution in the presence of only the covariate sex, the DIC value is given by 675.8 and assuming "model 3", that is, the bivariate geometric distribution in the presence of the covariates sex, age, presence of disease type GN, presence of disease type AN, presence of disease type PKD the DIC value is given by 668.2. This is, "model 3" gives better fit for the recurrence times of infection for kidney patients introduced by [15] (smaller value for DIC).

Observe that similar results were obtained by [19] assuming a bivariate exponential Block and Basu distribution for continuous lifetimes in the case considering only the covariate Sex. Some advantages of the bivariate geometric distribution: better convergence of Gibbs sampling algorithm even considering more non-informative prior distributions. 


\section{Concluding remarks}

The use of a discrete bivariate geometric distribution could be a new and good alternative for the analysis of bivariate lifetime data, especially under a Bayesian approach and using MCMC methods. Usually, in medical applications, we have the presence of censored data and the presence of one or more covariates, a situation that could bring us great difficulties to get the usual classical inferences for the parameters of the model like the convergence in the iterative numerical algorithm used to get the maximum likelihood estimators for the parameters of the model. It is also important to point out that these classical inference procedures are based on asymptotical normality of maximum likelihood estimators (see for example, Lawless,1982).This could be a problem when we have small data sizes or the presence of a large proportion of censored data, two situations very common in medical studies.Also it is important to point out that the use of available Bayesian software like the OpenBUGS software, only requires the specification of the distribution for the data and prior distributions for the parameters of the model, giving us a great simplification to obtain posterior summaries of interest, as it was observed in the application considering a medical data introduced in section 3.These results could be of great interest for applications in medical and engineering studies.

Appendix. Deviance Information Criterion (DIC)

The Deviance Information Criterion (DIC) is a criterion specially useful for selection models under the Bayesian approach where samples of the posterior distribution for the parameters of the model are obtained using MCMC methods.

The deviance is defined by,

$$
D(\theta)=-2 \log L(\theta)+c,
$$

where $\theta$ is a vector of unknown parameters of the model, $L(\theta)$ is the likelihood function of the model and $c$ is a constant that does not need to be known when the comparison between models is made.

The DIC criterion defined by [23] is given by,

$$
D I C=D(\hat{\theta})+2 n_{D}
$$

where $D(\hat{\theta})$ is the deviance evaluated at the posterior mean $\hat{\theta}=E(\theta \mid$ data $)$ and $n_{D}$ is the effective number of parameters of the model given by 
$n_{D}=\bar{D}-D(\hat{\theta})$, where $\bar{D}=E(D(\theta) \mid$ data $)$ is the posterior deviancemeasuring the quality of the data fit for the model. Smaller values of DIC indicates better models. Note that these values could be negative.

\section{References}

[1] J.A. Achcar and R.A. Leandro, Use of Markov Chain Monte Carlo methods in a Bayesian analysis of the Block and Basu bivariate exponential distribution, Annals of the Institute of Statistical Mathematics 50 (1998), pp. 403-416.

[2] B. Arnold, A characterization of the exponential distribution by multivariate geometric compounding. Sankhya 37 (1975), p.164-173.

[3] A.P. Basu and S. Dhar, Bivariate Geometric Distribution. Journal Applied Statistical Science 2(1) (1995), pp. 33-44.

[4] G. Casella and E.I. George, Explaining the Gibbs sampler, Amer. Statist. (1992), pp. 167-174.

[5] N. Davarzani and A. Parsian, Bayesian inference in dependent right censoring, Communications in Statistics - Theory and Methods 39 (2010), pp. 1270-1288.

[6] N. Davarzani and A. Parsian, Inference under right censoring in a discrete setup, Communications in Statistics - Theory and Methods 42 (2013), pp. 2362-2375.

[7] N. Davarzani, A. Parsian and R. Peeters, Dependent Right Censorship in the Marshall-Olkin Bivariate Weibull Distribution. Communications in Statistics - Theory and Methods,(2015), 44: 22222242.

[8] A.E. Gelfand and A.F.M. Smith, Sampling based approaches to calculating marginal densities, J. Amer. Statist. Assoc. (1990), pp. 398409.

[9] S.D., Grimshaw, J., McDonald, G. R., McQueen and S. Thorley, Estimating hazard functions for discrete lifetimes. Communications in Statistics, Part B-Simulation and Computation 34 (2005), pp. 451-463.

[10] D.D. Hanagal, Bivariate Weibull regression model based on censored samples. Statistical Papers 47 (2006), pp. 137-48.

[11] A. Jamalzadeh and D.Kundu, Weighted Marshall-Olkin bivariate exponential distribution. Statistics 47(5), (2013), pp 917-928 
[12] D. Kundu and Arjun K. Gupta, On bivariate Weibull Geometric distribution, Journal of Multivariate Analysis 123(1), (2014), pp. 19-29.

[13] J.F. Lawless, Statistical Models and Methods for Lifetime Data,Wiley, NewYork, (1982).

[14] A.W. Marshall and I. Olkin, A generalized bivariate exponential distribution, J. Appl. Probab. (1967), pp. 291-302.

[15] C.A. McGilchrist and C.W. Aisbett, Regression with frailty in survival analysis, Biometrics (1991), pp. 461-466.

[16] K.R.M. Nair and U. Nair, On characterizing the bivariate exponential and geometric distributions. Annals of the Institute of Statistical Mathematics, 40(2) (1988), pp.267-271.

[17] S. Nandi and I., Dewan, An EM algorithm for estimating the parameters of bivariate Weibull distribution under random censoring. Computational Statistics and Data Analysis 54 (2010), pp. 1559-1569.

[18] P.G. Sankaran and D. Kundu, A bivariate Pareto model. Statistics 48(2), (2014), pp. 241-255.

[19] C.A. Santos and J.A. Achcar, A Bayesian analysis for the Block and Basu bivariate exponential distribution in the presence of covariates and censored data, Journal of Applied Statistics 38 (2011), pp. 22132223.

[20] S.K. Sarkar, A continuous bivariate exponential distribution, J. Amer. Statist. Assoc. (1987), pp. 667-675.

[21] A.M. Sarhan and N. Balakrishnan, A new class of bivariate distribution, Journal of Multivariate Analysis 98 (2007), pp. 1508-1527

[22] Shih JH. Modeling multivariate discrete failure time data. Biometrics 54 (1998):1115-1128.

[23] D.J. Spiegelhalter, N.G. Best, B.P. Carlin, and A. LindeVan der, Bayesian measures of model complexity and fit (with discussion), Journal of the Royal Statistical Society B (2002), pp. 583-639.

Received August, 2015 